\title{
Asymptotic Behavior of Nonlinear Transmission Plate Problem
}

\author{
Mykhailo Potomkin \\ B.Verkin Institute for Low Temperature Physics and Engineering of NASU, \\ 47 Lenin Ave., Kharkov 61103 , Ukraine \\ mika_potemkin@mail.ru \\ http://ilt.kharkov.ua
}

\begin{abstract}
We study a nonlinear transmission problem for a plate which consists of thermoelastic and isothermal parts. The problem generates a dynamical system in a suitable Hilbert space. Main result is the proof of asymptotic smoothness of this dynamical system and existence of a compact global attractor in special cases.
\end{abstract}

Keywords: Transmission problems, long-time behavior, asymptotic smoothness, attractor.

\section{Introduction}

In this work we deal with a partially thermoelastic plate: one part is of isothermal material, the second one is of material whose structure does not neglect the thermal dissipation. Due to the thermal dissipation, purely thermoelastic plate is exponentially stable in linear case (see, e.g., survey in [1, Chapter 3A]) or possesses a compact global attractor in cases of different kind of nonlinearities (see, e.g., [2]). On the other hand, in the case of purely isothermal plate the energy is constant, thus there could not be any decay to zero point in the linear model and global attractor in the nonlinear model. Here we investigate whether the thermal dissipation on a part of the plate is enough to have any stabilization. Exponential stability of the linear problem of this type was established in [3].

Let $\Omega_{1}, \Omega_{2}$ and $\Omega$ be bounded open sets in $\mathbb{R}^{2}, \Gamma_{0}=\bar{\Omega}_{1} \cap \bar{\Omega}_{2}, \Gamma_{1}=\partial \Omega_{1} / \Gamma_{0}$ and $\Gamma_{2}=\partial \Omega_{2} / \Gamma_{0}$ be smooth surfaces. We also assume that $\Omega=\Omega_{1} \cup \Omega_{2} \cup \Gamma_{0}$ and $\bar{\Gamma}_{1} \cap \bar{\Gamma}_{2}=\emptyset$. In the model under consideration the plate (its middle surface), in equilibrium, occupies the domain $\Omega$ which consists of two parts $\Omega_{1}$ and $\Omega_{2}$ with common boundary $\Gamma_{0}$. In what follows below $\nu$ denotes the outward normal vector on $\Gamma_{1}$ and $\Gamma_{2}$, in cases of common boundary $\Gamma_{0}$ the vector $\nu$ is outward to $\Omega_{2}$.

We consider the following system of equations:

$$
\begin{aligned}
\rho_{1} u_{t t}+\beta_{1} \Delta^{2} u+\mu \Delta \theta+F_{1}(u, v)=0 & \text { in } \Omega_{1} \times \mathbb{R}^{+}, \\
\rho_{0} \theta_{t}-\beta_{0} \Delta \theta-\mu \Delta u_{t}=0 & \text { in } \Omega_{1} \times \mathbb{R}^{+}, \\
\rho_{2} v_{t t}+\beta_{2} \Delta^{2} v+F_{2}(u, v)=0 & \text { in } \Omega_{2} \times \mathbb{R}^{+} .
\end{aligned}
$$

D. Hömberg and F. Tröltzsch (Eds.): CSMO 2011, IFIP AICT 391, pp. 521 527, 2013.

(C) IFIP International Federation for Information Processing 2013 


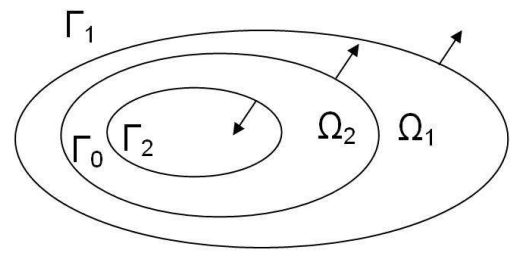

Fig. 1.

We impose the following boundary conditions:

$$
\begin{aligned}
& u=\frac{\partial u}{\partial \nu}=0 \text { on } \Gamma_{1}, \quad v=\frac{\partial v}{\partial \nu}=0 \text { on } \Gamma_{2}, \\
& u=v, \frac{\partial u}{\partial \nu}=\frac{\partial v}{\partial \nu}, \beta_{1} \Delta u=\beta_{2} \Delta v, \beta_{1} \frac{\partial \Delta u}{\partial \nu}+\mu \frac{\partial \theta}{\partial \nu}=\beta_{2} \frac{\partial \Delta v}{\partial \nu} \text { on } \Gamma_{0}, \\
& \theta=0 \text { on } \Gamma_{0}, \quad \frac{\partial \theta}{\partial \nu}+\lambda \theta=0 \text { on } \Gamma_{1} .
\end{aligned}
$$

The equations above are equipped with the following initial data:

$$
\begin{aligned}
& u(\mathbf{x}, 0)=u_{0}(\mathbf{x}), \quad u_{t}(\mathbf{x}, 0)=u_{1}(\mathbf{x}), \quad \theta(\mathbf{x}, 0)=\theta_{0}(\mathbf{x}) \text { in } \Omega_{1}, \\
& v(\mathbf{x}, 0)=v_{0}(\mathbf{x}), \quad v_{t}(\mathbf{x}, 0)=v_{1}(\mathbf{x}) \text { in } \Omega_{2} .
\end{aligned}
$$

Coefficients $\rho_{i}, \beta_{i}$ and $\mu$ are strictly positive, the functions

$$
F_{i}: H^{2}\left(\Omega_{1}\right) \times H^{2}\left(\Omega_{2}\right) \longrightarrow L^{2}\left(\Omega_{i}\right), i=1,2
$$

are nonlinear.

Functions $u(\mathbf{x}, t)$ and $v(\mathbf{x}, t)$ describe the vertical displacement of the plate in $\Omega_{1}$ and $\Omega_{2}$, respectively, and function $\theta(\mathbf{x}, t)$ describes the temperature regime. Equations (1) and (3) are plate equations, equation (1) is a heat equation. Equalities in (4) mean that the plate is clamped along $\Gamma_{1}$ and $\Gamma_{2}$. Boundary conditions (5) are transmission boundary conditions. Temperature function $\theta$ satisfies the Newton law of cooling on $\Gamma_{1}$ with some positive coefficient $\lambda$ and vanishes on $\Gamma_{0}$ (see equalities (6) ).

Let us introduce four problems which are concrete examples of abstract problem (11)-(7).

Problem A corresponds to oscillations of a plate in the Berger approach. In this case

$$
F_{1}(u, v)=-M(u, v) \Delta u, \quad F_{2}(u, v)=-M(u, v) \Delta v,
$$

where

$$
M(u, v)=\Gamma+\gamma\left[\int_{\Omega_{1}}|\nabla u|^{2} \mathrm{~d} \mathbf{x}+\int_{\Omega_{2}}|\nabla v|^{2} \mathrm{~d} \mathbf{x}\right] .
$$

Here $\Gamma$ is a real number, $\gamma$ is strictly positive. 
In problem B we consider scalar nonlinearities, namely,

$$
F_{1}(u, v)=f_{1}(u), \quad F_{2}(u, v)=f_{2}(v),
$$

where the scalar functions $f_{i} \in C^{2}$ satisfy the following conditions: there exist such $p>1$ and $C>0$ that

$$
\begin{aligned}
& \left|f_{i}^{\prime}(s)\right| \leq C\left(1+|s|^{p}\right), \\
& \liminf _{|s| \rightarrow \infty} \frac{f_{i}(s)}{s}>0 .
\end{aligned}
$$

In problem $\mathbf{C}$ we deal with the von Karman nonlinearity. Here we set $\Gamma_{2}=\emptyset$ and

$$
F_{1}(u, v)=-\left[u, \mathcal{F}_{1}\right], \quad F_{2}(u, v)=-\left[v, \mathcal{F}_{2}\right],
$$

where $[\psi, \varphi]=\psi_{x x} \varphi_{y y}+\psi_{y y} \varphi_{x x}-2 \psi_{x y} \varphi_{x y}$ is the von Karman brackets; the Airy stress functions $\mathcal{F}_{1}$ and $\mathcal{F}_{2}$ solve the following equations (parameters $\gamma_{i}$ are strictly positive):

$$
\gamma_{1} \Delta^{2} \mathcal{F}_{1}+[u, u]=0 \text { in } \Omega_{1} \times \mathbb{R}^{+} \text {and } \gamma_{2} \Delta^{2} \mathcal{F}_{2}+[v, v]=0 \text { in } \Omega_{2} \times \mathbb{R}^{+}
$$

with the boundary conditions:

$$
\begin{aligned}
& \text { on } \Gamma_{1}: \mathcal{F}_{1}=\frac{\partial}{\partial \nu} \mathcal{F}_{1}=0, \\
& \text { on } \Gamma_{0}: \mathcal{F}_{1}=\mathcal{F}_{2}, \quad \frac{\partial}{\partial \nu} \mathcal{F}_{1}=\frac{\partial}{\partial \nu} \mathcal{F}_{2}, \quad \gamma_{1} \Delta \mathcal{F}_{1}=\gamma_{2} \Delta \mathcal{F}_{2}, \gamma_{1} \frac{\partial}{\partial \nu} \Delta \mathcal{F}_{1}=\gamma_{2} \frac{\partial}{\partial \nu} \Delta \mathcal{F}_{2}
\end{aligned}
$$

Problem $\mathbf{D}$ corresponds to the problem of oscillations of the Berger plate on an elastic base. Mathematically, this problem is a a generalization of problems $\mathrm{A}$ and $\mathrm{B}$. Nonlinearities $F_{i}$ are given by the following equalities:

$$
\begin{aligned}
& F_{1}(u, v)=-M\left(\|\nabla u\|_{\Omega_{1}}^{2}+\|\nabla v\|_{\Omega_{2}}^{2}\right) \Delta u+a_{1}(\mathbf{x}) u|u|^{p-1}+g_{1}(\mathbf{x}, u), \\
& F_{2}(u, v)=-M\left(\|\nabla u\|_{\Omega_{1}}^{2}+\|\nabla v\|_{\Omega_{2}}^{2}\right) \Delta v+a_{2}(\mathbf{x}) v|v|^{p-1}+g_{2}(\mathbf{x}, v),
\end{aligned}
$$

where $M(s)=s^{1+\alpha}$ with $\alpha>0, a_{1}(\mathbf{x}) \in L^{\infty}\left(\Omega_{1}\right)$ and $a_{2}(\mathbf{x}) \in L^{\infty}\left(\Omega_{2}\right)$. We assume that the following condition holds:

$$
\text { either } a(\mathbf{x}) \geq c_{0} \forall \mathbf{x} \in \Omega \text { or } 2(\alpha+2)>p+1, p \geq 1 .
$$

Here $a=\left\{a_{1}, a_{2}\right\}$ and $c_{0}>0$ is a small number. The functions $g_{1}(\mathbf{x}, u)$ and $g_{2}(\mathbf{x}, v)$ are scalar and satisfy the growth condition for some $\varepsilon_{0}>0$ and any $\mathbf{x}_{i} \in \Omega_{i}$ :

$$
\left|\frac{\partial}{\partial u} g_{1}\left(\mathbf{x}_{1}, u\right)\right|+\left|\frac{\partial}{\partial v} g_{2}\left(\mathbf{x}_{2}, v\right)\right| \leq C\left(1+|u|^{\max \left\{0, p-1-\varepsilon_{0}\right\}}+|v|^{\max \left\{0, p-1-\varepsilon_{0}\right\}}\right),
$$

Our main result is the property of asymptotic smoothness of the dynamical system generated by weak solutions of problem (11)-(7). To achieve it we use method of so-called compensated compactness function first introduced in [7] (see 
also [2, Proposition 2.10]). The method for various types of nonlinearities was developed in 2. We also need to impose the following conditions on parameters:

$$
\rho_{1} \geq \rho_{2} \text { and } \beta_{1} \leq \beta_{2}
$$

and on geometric structure of $\Omega_{i}$ :

$$
\begin{aligned}
& \left(\mathbf{x}-\mathbf{x}_{0}\right) \cdot \nu(\mathbf{x}) \geq \delta_{0} \text { on } \Gamma_{0}, \\
& \left(\mathbf{x}-\mathbf{x}_{0}\right) \cdot \nu(\mathbf{x}) \leq 0 \text { on } \Gamma_{2}
\end{aligned}
$$

for some $\mathbf{x}_{0} \in \mathbb{R}^{2}$ and $\delta_{0}>0$. Imposing these conditions authors of work 3 ] proved the exponential stability of linear problem $\left(F_{1}=F_{2}=0\right)$.

Asymptotic smoothness is important property of a dynamical system if one wants to prove the existence of a compact global attractor. In particular, asymptotically smooth dynamical system possesses a compact global attractor, if it possesses an appropriate Lyapunov function (for details we refer to [2, Chapter 2.4]).

Our result on asymptotic smoothness is applicable for each of the concrete problem listed above. For problems A, B and D we proved that the corresponding dynamical system possesses an appropriate Lyapunov function and, thus, there exists a compact global attractor.

Up to our best knowledge asymptotic behavior in transmission problem for a plate of types A, B, C and D was not considered before.

One can find formulations of our results in the next section. For proofs and other details we refer to our works [4] and [5].

\section{Formulation of Main Result}

\subsection{Dynamical System}

Below notation $\psi=\left\{\psi_{1}, \psi_{2}\right\}$ means that $\psi(\mathbf{x})$ defined for $\mathbf{x} \in \Omega$ is equal to $\psi_{i}(\mathbf{x})$, if $\mathbf{x} \in \Omega_{i}$, for $i=1,2$.

To formulate a well-posedness result we need to impose the following conditions:

$$
\begin{array}{r}
\int_{\Omega_{1}}\left|F_{1}\left(w_{1}^{1}, w_{2}^{1}\right)-F_{1}\left(w_{1}^{2}, w_{2}^{2}\right)\right|^{2} \mathrm{~d} \mathbf{x}+\int_{\Omega_{2}}\left|F_{2}\left(w_{1}^{1}, w_{2}^{1}\right)-F_{2}\left(w_{1}^{2}, w_{2}^{2}\right)\right|^{2} \mathrm{~d} \mathbf{x} \\
\leq C(r)\left\|\left\{w_{1}^{1}-w_{1}^{2}, w_{2}^{1}-w_{2}^{2}\right\}\right\|_{H_{T}^{2}}^{2}
\end{array}
$$

for all $\left\|\left\{w_{1}^{i}, w_{2}^{i}\right\}\right\|_{H_{0}^{2}(\Omega)} \leq r, i=1,2$. We also assume that there exists such continuous functional $\Pi: H_{0}^{2}(\Omega) \rightarrow \mathbb{R}$ that

$$
\begin{aligned}
& \frac{\mathrm{d}}{\mathrm{d} t} \Pi\left(w_{1}, w_{2}\right)=\int_{\Omega_{1}} F_{1}\left(w_{1}, w_{2}\right) w_{1, t} \mathrm{~d} \mathbf{x}+\int_{\Omega_{2}} F_{2}\left(w_{1}, w_{2}\right) w_{2, t} \mathrm{~d} \mathbf{x}, \\
& \Pi\left(w_{1}, w_{2}\right) \geq-C, \quad \exists C>0, \\
& \Pi\left(w_{1}, w_{2}\right) \leq \mathcal{G}\left(\left\|\left\{w_{1}, w_{2}\right\}\right\|_{H_{0}^{2}(\Omega)}\right) .
\end{aligned}
$$


Condition (16) holds for

$$
\left\{w_{1}, w_{2}\right\} \in L^{2}\left(0, T ; H_{0}^{2}(\Omega)\right) \text { and }\left\{w_{1, t}, w_{2, t}\right\} \in L^{2}((0, T) \times \Omega) .
$$

Conditions (17) and (18) hold for all $\left\{w_{1}, w_{2}\right\} \in H_{0}^{2}(\Omega)$. The scalar function $\mathcal{G}: \mathbb{R}^{+} \longrightarrow \mathbb{R}^{+}$is supposed to be bounded on bounded intervals. The condition (16) also means that feedback forces $\left\{F_{1}(u, v), F_{2}(u, v)\right\}$ are potential, i.e., $\left\{F_{1}(u, v), F_{2}(u, v)\right\}$ is a Freshet derivative of $\Pi(u, v)$.

For problem A $\Pi\left(w_{1}, w_{2}\right)=\frac{1}{4} M^{2}\left(w_{1}, w_{2}\right)$, for problem B:

$$
\Pi\left(w_{1}, w_{2}\right)=\int_{\Omega_{1}} \int_{0}^{w_{1}(x)} f_{1}(s) \mathrm{d} s \mathrm{~d} \mathbf{x}+\int_{\Omega_{1}} \int_{0}^{w_{2}(x)} f_{2}(s) \mathrm{d} s \mathrm{~d} \mathbf{x} .
$$

For problem $\mathrm{C}$, if calculate $\mathcal{F}_{i}$ according to (9):

$$
\Pi\left(w_{1}, w_{2}\right)=\frac{\gamma_{1}}{2} \int_{\Omega_{1}}\left|\Delta \mathcal{F}_{1}\right|^{2} \mathrm{~d} \mathbf{x}+\frac{\gamma_{2}}{2} \int_{\Omega_{2}}\left|\Delta \mathcal{F}_{2}\right|^{2} \mathrm{~d} \mathbf{x}
$$

For problem D:

$$
\Pi(w)=\frac{1}{2(\alpha+2)}\|\nabla w\|_{L^{2}(\Omega)}^{2(\alpha+2)}+\frac{1}{p+1} \int_{\Omega} a(\mathbf{x})|w(\mathbf{x})|^{p+1} d \mathbf{x}+\int_{\Omega} \int_{0}^{w(\mathbf{x})} g(\mathbf{x}, s) d s d \mathbf{x}
$$

where $a=\left\{a_{1}, a_{2}\right\}$ and $g=\left\{g_{1}, g_{2}\right\}$.

We introduce phase space $\mathcal{H}=H_{0}^{2}(\Omega) \times L^{2}(\Omega) \times L^{2}\left(\Omega_{2}\right)$ and energy function $\mathcal{E}: \mathcal{H} \longrightarrow \mathbb{R}$ which we define for an argument $w=\left(w_{1}, w_{2}, w_{3}, w_{4}, w_{5}\right)$ (here $\left\{w_{1}, w_{2}\right\} \in H_{0}^{2}(\Omega),\left\{w_{3}, w_{4}\right\} \in L^{2}(\Omega)$ and $\left.w_{5} \in L^{2}(\Omega)\right)$ as follows

$$
\begin{array}{r}
\mathcal{E}(w)=\frac{1}{2}\left[\int_{\Omega_{1}} \beta_{1}\left|\Delta w_{1}\right|^{2}+\rho_{1}\left|w_{3}\right|^{2}+\rho_{0}\left|w_{5}\right|^{2} d \mathbf{x}\right. \\
\left.+\int_{\Omega_{2}} \beta_{2}\left|\Delta w_{2}\right|^{2}+\rho_{2}\left|w_{4}\right|^{2} d \mathbf{x}+2 \Pi\left(w_{1}, w_{2}\right)\right] .
\end{array}
$$

Now we are in position to formulate the theorem on well-posedness of problem (11)-(7) .

Theorem 1. Let (15), (16), 117) and (18) hold. Then for any initial $w_{0} \in \mathcal{H}$ and $T>0$ there exists a unique weak solution $w(t) \in C([0, T] ; \mathcal{H})$. Moreover, it satisfies the energy equality:

$$
\mathcal{E}(w(T))-\mathcal{E}(w(t))=-\int_{t}^{T} \int_{\Omega_{1}} \beta_{0}\left|\nabla w_{5}\right|^{2} d \mathbf{x} d \tau-\int_{t}^{T} \int_{\Gamma_{1}} \beta_{0} \lambda\left|w_{5}\right|^{2} d \Gamma d \tau
$$

for all $0 \leq t \leq T$. If one sets $S_{t} w_{0}=w(t)$, then $\left(\mathcal{H}, S_{t}\right)$ is a continuous dynamical system. 


\section{$2.2 \quad$ Asymptotic Smoothness}

In this subsection we define the notion of asymptotic smoothness of a dynamical system, impose additional conditions on nonlinear functions $F_{i}$ and formulate our result on asymptotic smoothness of the dynamical system $\left(\mathcal{H}, S_{t}\right)$.

Definition 1. Let $\left(X, S_{t}\right)$ be a dynamical system. Assume that $X$ is a complete metric space and $S_{t}$ is a semigroup of operators on $X$.The dynamical system $\left(X, S_{t}\right)$ is said to be asymptotically smooth if for any positively invariant bounded set $D \subset X$ there exists a compact $K$ in the closure $\bar{D}$ of $D$ such that

$$
\lim _{t \rightarrow+\infty} \sup _{x \in D} \operatorname{dist}_{X}\left(S_{t} x, K\right)=0 .
$$

For the detailed discussion and applications of asymptotic smoothness we refer to, e.g., 6] and [2].

To obtain asymptotic smoothness we need to impose additional conditions on nonlinear functions $F_{i}$.

Assume that there exist such $\delta, \sigma>0$ that

$$
\begin{gathered}
w \longmapsto \Pi(w): H^{2-\delta} \longrightarrow \mathbb{R} \text { is a continuous mapping, } \\
w \longmapsto \Pi_{\Phi}^{\prime}(w): H^{2-\delta} \longrightarrow H^{-\sigma} \text { is a continuous mapping. }
\end{gathered}
$$

Our main result is the following theorem.

Theorem 2. Let (12), (13), 14), (15), 17), (18), (22) and (23) hold. Then the dynamical system $\left(\mathcal{H}, S_{t}\right)$ is asymptotically smooth.

The method of the proof is based on idea of compensated compactness function (see 7 and 2]). This result is applicable for all concrete problems, A,B,C and $\mathrm{D}$, listed in introduction.

\subsection{Compact Global Attractor and Its Properties}

The direct application of [2, Corollary 2.29] gives the following theorem.

Theorem 3. Let conditions of theorem 2 hold. Assume also that for all solution $w(t)$ to problem (1)-(7) the following statement holds:

if $\mathcal{E}(w(t))$ does not depend on $t$, then $w(t)$ does not depend on $t$.

In other words, energy $\mathcal{E}$ is constant only on stationary trajectories.

Then the dynamical system $\left(\mathcal{H}, S_{t}\right)$ possesses a compact global attractor.

Verification of condition (24) could be reduced to the problem which is relative to unique continuation problems. Let $w(t)=\left(u(t), v(t), u_{t}(t), v_{t}(t), \theta(t)\right)$ be a solution of problem (1)-(7) such that $\mathcal{E}(w(t))$ is constant. Let us denote $v^{h}(t):=$ $v(t+h)-v(t), B(t) v^{h}:=F_{2}(u, v(t+h))-F_{2}(u, v(t))$ for some $h>0$. If $\mathcal{E}(w(t))$ does not depend on $t$, then $v^{h}$ solves the following problem:

$$
\begin{aligned}
& \rho_{2} v_{t t}^{h}+\beta_{2} \Delta^{2} v^{h}=B(t) v^{h}, \\
& \left.v^{h}\right|_{\Gamma_{0}}=\left.\frac{\partial v^{h}}{\partial \nu}\right|_{\Gamma_{0}}=\left.\Delta v^{h}\right|_{\Gamma_{0}}=\left.\frac{\partial \Delta v^{h}}{\partial \nu}\right|_{\Gamma_{0}}=0 .
\end{aligned}
$$


Therefore, if we prove that $v^{h}(t) \equiv 0$, then we will have that $w(t) \equiv w_{0}$.

Using Pochozhaev multiplicator $\left(\mathbf{x}-\mathbf{x}_{0}\right) \cdot \nabla v^{h}$, where vector $\mathbf{x}_{0}$ is the same as in conditions (13) and (14), for concrete problems $\mathrm{A}$ and $\mathrm{B}$ with $f_{2} \equiv 0$ and Carleman estimates obtained in [8] for concrete problems B and D, we manage to verify (24). Thus, we obtain the following corollary.

Corollary 1. Let (12), (13), 14) hold. The dynamical system $\left(\mathcal{H}, S_{t}\right)$ corresponding to one of the concrete problems $A, B$ or $D$ possesses a compact global attractor.

\section{References}

1. Lasiecka, I., Triggiani, R.: Control Theory for PDEs, vol. 1. Cambridge University Press, Cambridge (2000)

2. Chueshov, I.D., Lasiecka, I.: Long-time behavior of second order evolution equations with nonlinear damping. Memoirs of AMS, vol. (912). Americal Mathematical Society, Providence (2008)

3. Rivera, J.E.M., Oquendo, H.P.: A transmission problem for thermoelastic plates. Quarterly of Applied Mathematics 62(2), 273-293 (2004)

4. Potomkin, M.: A nonlinear transmission problem for a compound plate with thermoelastic part (2010), http://arxiv.org/abs/1003.3332

5. Potomkin, M.: On Transmission Problem for Berger Plates on an Elastic Base. Journal of Mathematical Physics, Analysis and Geometry 7(1), 96-102 (2011)

6. Raugel, G.: Global attractors in partial differential equations. In: Fiedler, B. (ed.) Handbook of Dynamical Systems, vol. 2, pp. 885-982. Elsevier, Amsterdam (2002)

7. Khanmamedov, A.: Global attractors for von Karman equations with nonlinear dissipation. J. Math. Anal. Appl. 318, 92-101 (2006)

8. Albano, P.: Carleman estimates for the Euler-Bernoulli plate operator. Electronic Journal of Diff. Eq. 53, 1-13 (2000) 